\title{
CONFÉRENCE
}

\section{Nouvelles technologies : laser et chirurgie buccale}

\author{
Gianluigi Caccianiga \\ Département de Chirurgie et de Médecine Orales, Université de Milan Bicocca. Milan - Italie \\ glcaccianiga@hotmail.it
}

Seules les connaissances des propriétés physiques et des longueurs d'onde disponibles peuvent permettre au praticien d'utiliser des systèmes lasers appropriés à la chirurgie buccale.

Si l'usage du laser combiné aux méthodes thérapeutiques non chirurgicales conventionnelles dans les traitements parodontaux, est destiné à améliorer les résultats thérapeutiques, le laser peut aussi, en raison de ses capacités antibactériennes et de ses propriétés bio-stimulantes, acquérir le titre de technique de choix en chirurgie buccale et en implantologie orale.

En effet, différentes études scientifiques in vivo et in vitro ont démontré l'aptitude de la thérapie par laser à améliorer les résultats obtenus par les procédures de chirurgie buccale traditionnelles en particulier lors de l'association du laser aux agents antibactériens tel que le péroxyde d'hydrogène, la chlorhexidine ou les solutions à base d'iode. L'utilisation du laser dans les chirurgies avec lambeaux permet une activité antibactérienne plus en profondeur et plus persistante aussi bien au sein des tissus mous que des tissus durs, tels que les tissus périimplantaires et osseux infiltrés permettant ainsi une meilleure préparation des sites à recevoir des matériaux de restauration.

Il a aussi été démontré que la biostimulation par laser du tissu osseux durant et après chirurgie implantaire non seulement favorise la formation osseuse péri-implantaire mais permet aussi celle-ci dans des délais plus courts. Enfin, l'usage du laser peut-être envisagé dans les reconstructions pré-implantaires chirurgicales (greffes osseuses, implantation immédiate, sinus-lift) avec ou sans membrane ou PRF/PRP. 